\title{
SYMBOL-BASED PRECONDITIONING FOR RIESZ DISTRIBUTED-ORDER SPACE-FRACTIONAL DIFFUSION EQUATIONS*
}

\author{
MARIAROSA MAZZA ${ }^{\dagger}$, STEFANO SERRA-CAPIZZANO ${ }^{\dagger}$, AND MUHAMMAD USMAN ${ }^{\ddagger}$
}

\begin{abstract}
In this work, we examine the numerical solution of a 1D distributed-order space-fractional diffusion equation. Discretizing the given problem by means of an implicit finite difference scheme based on the shifted Grünwald-Letnikov formula, the resulting linear systems show a Toeplitz structure. Then, by using well-known spectral tools for Toeplitz sequences, we determine the corresponding symbol describing its asymptotic eigenvalue distribution as the matrix size diverges. The spectral analysis is performed under different assumptions with the aim of estimating the intrinsic asymptotic ill-conditioning of the involved matrices. The obtained results suggest to precondition the involved linear systems with either a Laplacian-like preconditioner or with more general $\tau$ preconditioners. Due to the symmetric positive definite nature of the coefficient matrices, we opt for the preconditioned conjugate gradient method, and we compare the performances of our proposal with a Strang circulant alternative given in the literature.
\end{abstract}

Key words. fractional diffusion equations, Toeplitz matrices, spectral distribution, preconditioning

AMS subject classifications. 35R11, 15B05, 15A18, 65F08

1. Introduction. In the last decades, constant-order fractional diffusion equations (FDEs) have been widely studied and successfully applied for modeling anomalous diffusion in a wide range of applicative fields like, e.g., finance, biology, image processing, and plasma physics $[2,9,17,20]$. The reason why this research field is so fruitful essentially relies on the fact that a proper noninteger parameter, the so-called fractional derivative order, can be tuned in order to model enhanced diffusivity not covered by classical differential modeling. An even more powerful tool in this framework is represented by distributed-order operators, which are particularly effective for depicting anomalous diffusion with combined scaling exponents. In [6], Caputo firstly proposed the use of distributed-order fractional operators to describe the stress-strain relations of inelastic media. Later, the distributed-order fractional differential equations were also applied to diffusion processes; see, e.g., [5].

As for constant-order FDEs, also for distributed-order FDEs analytical solutions are rarely available, and numerical methods for the computation of approximate solutions need to be investigated. Fan and Liu [10] proposed a finite element method for solving distributedorder FDEs on an irregular convex domain. A finite volume method for the distributed-order advection-diffusion equation has been described by Li et al. [18], and an implicit finite difference scheme has been used in [1].

However, as for constant-order FDEs, those methods usually produce full coefficient matrices, which require $\mathcal{O}\left(n^{3}\right)$ computational costs and $\mathcal{O}\left(n^{2}\right)$ storage costs using general purpose direct methods, where $n$ is the number of grid points. On the other hand, in presence of uniform gridding, the shift-invariant nature of the underlying operators leads to Toeplitz-like matrices, and then the storage requirement can be reduced from $\mathcal{O}\left(n^{2}\right)$ to $\mathcal{O}(n)$ and the complexity of the matrix-vector product from $\mathcal{O}\left(n^{2}\right)$ to $\mathcal{O}(n \log n)$, thanks to the use of the fast Fourier transform (FFT). This is the approach that has been adopted in [16], where the author proposed to use the conjugate gradient (CG) method combined with a Strang circulant preconditioner.

\footnotetext{
*Received December 3, 2020. Accepted June 21, 2021. Published online on August 10, 2021. Recommended by Maya Neycheva.

${ }^{\dagger}$ Department of Humanities and Innovation, University of Insubria, Via Valleggio 11, Como, Italy ( $\{$ mariarosa.mazza, s.serracapizzano\} @uninsubria.it).

${ }^{\ddagger}$ Department of Science and High Technology, University of Insubria, Via Valleggio 11, Como, Italy (musman2@uninsubria.it).
} 
Mimicking the analysis for constant-order FDEs performed in [7, 8, 19], in the present paper we focus on the spectral study of the symmetric positive definite coefficient matrixsequences given in [16], and we compute the associated symbol, which represents the distribution of the eigenvalues as the matrix size diverges. We remind that the evaluation of the symbol over uniform equispaced grids in the domain leads to a reasonable approximation of the eigenvalues when the matrix size is sufficiently large. The spectral analysis is discussed under different assumptions with the aim of estimating the intrinsic asymptotic ill-conditioning of the involved matrices. The obtained results suggest to precondition the involved linear systems with a Laplacian-like preconditioner. Indeed, it turns out that the overall symbol associated to our matrix structures behaves as $\theta^{\alpha_{\ell}}$ in a neighborhood of $\theta=0$, where $\alpha_{\ell} \rightarrow 2$ as $\ell \rightarrow \infty$, with $\ell$ being the number of uniform subintervals in $(1,2)$ that we choose for the quadrature. This is the technical reason why we propose the discrete Laplacian as main ingredient of a preconditioning strategy since the symbol of the discrete Laplacian, $2-2 \cos (\theta)$, behaves as $\theta^{2}$ in a neighborhood of $\theta=0$ (see $[4,22,23]$ for a theoretical explanation).

Furthermore, we compare the performances of the proposed Laplacian-like preconditioner with the Strang circulant preconditioner proposed in [16]. We stress that the linear systems associated with our proposal can be solved optimally in $\mathcal{O}(n)$ operations by the standard Gaussian Elimination (known also as Thomas algorithm when applied to banded matrices), which does not ask for "all-to-all communications" as in case of the FFT algorithm. We observe that the discrete Laplacian belongs to the $\tau$-algebra and hence also more general $\tau$-preconditioners are considered and tested numerically. As described in [24], for symmetric Toeplitz matrices with real coefficients, the (real) $\tau$-algebra allows one to obtain approximations of the original coefficient matrix showing a correction with a slightly lower rank when compared with the classical circulant algebra: this slightly smaller rank correction is reflected in a slightly smaller number of preconditioned conjugate gradient (PCG) iterations as reported in the numerical test section.

The paper is organized as follows. Section 2 is devoted to the presentation of the continuous problem, its numerical approximation, and the basic features of the resulting coefficient matrices. Section 3 contains the spectral and conditioning analysis mainly based on the Toeplitz technology and its application to the spectral study of the resulting preconditioned matrices. Finally in Section 4 we present and discuss the numerical results, and in Section 5 we draw conclusions by emphasizing the relevance of the theoretical results and a few open problems with special attention to the higher-dimensional setting and to stability issues.

2. Riesz distributed-order space-fractional diffusion equations and finite difference approximation. In this paper, we study the following initial-boundary value problem (see [1]):

$$
\left\{\begin{aligned}
\frac{\partial u(x, t)}{\partial t} & =\int_{1}^{2} \rho(\alpha) \frac{\partial^{\alpha} u(x, t)}{\partial|x|^{\alpha}} d \alpha+f(x, t), & & (x, t) \in \Omega=[a, b] \times[0, T] \\
u(x, 0) & =u_{0}(x), & & x \in(a, b), \\
u(a, t) & =u(b, t)=0, & & t \in(0, T]
\end{aligned}\right.
$$

where $\rho(\alpha)$ is the kernel function, which satisfies

$$
\rho(\alpha) \geq 0, \quad 0<\int_{1}^{2} \rho(\alpha) c(\alpha)<\infty,
$$

while $f(x, t)$ is the source term and $\frac{\partial^{\alpha} u(x, t)}{\partial|x|^{\alpha}}$ is the Riesz fractional derivative of order $1<\alpha<2$ with respect to $x$ defined as

$$
\frac{\partial^{\alpha} u(x, t)}{\partial|x|^{\alpha}}=c(\alpha)\left({ }_{a} D_{x}^{\alpha} u(x, t)+{ }_{x} D_{b}^{\alpha} u(x, t)\right), \quad c(\alpha)=\frac{-1}{2 \cos \left(\frac{\alpha \pi}{2}\right)}>0 .
$$


The left-sided and right-sided Riemann-Liouville fractional derivatives ${ }_{a} D_{x}^{\alpha} u(x, t),{ }_{x} D_{b}^{\alpha} u(x, t)$ are in turn defined as

$$
\begin{aligned}
& { }_{a} D_{x}^{\alpha} u(x, t)=\frac{1}{\Gamma(2-\alpha)} \frac{d^{2}}{d x^{2}} \int_{a}^{x}(x-y)^{1-\alpha} u(y, t) d y, \\
& { }_{x} D_{b}^{\alpha} u(x, t)=\frac{1}{\Gamma(2-\alpha)} \frac{d^{2}}{d x^{2}} \int_{x}^{b}(y-x)^{1-\alpha} u(y, t) d y,
\end{aligned}
$$

where $\Gamma(\cdot)$ is the gamma function. As done in [1], we adopt a second-order finite difference method to discretize equation (2.1). Let $n$ and $m$ be positive integers, $h=\frac{b-a}{n+1}$ be the spatial width, $\Delta t=\frac{T}{m}$ be the time step-size, and consider the following partition

$$
\begin{array}{ll}
x_{i}=a+i h, & i=0,1, \ldots, n+1, \\
t_{j}=j \Delta t, & j=0,1, \ldots, m .
\end{array}
$$

In order to discretize the left and right Riemann-Liouville fractional derivatives in space, we exploit the weighted and shifted Grünwald-Letnikov difference scheme given in [15], i.e.,

$$
\begin{aligned}
& { }_{a} D_{x}^{\alpha} u\left(x_{i}, t\right)=\frac{1}{h^{\alpha}} \sum_{q=0}^{i} \omega_{q}^{(\alpha)} u\left(x_{i-q+1}, t\right)+\mathcal{O}\left(h^{2}\right), \\
& { }_{x} D_{b}^{\alpha} u\left(x_{i}, t\right)=\frac{1}{h^{\alpha}} \sum_{q=0}^{n-i+1} \omega_{q}^{(\alpha)} u\left(x_{i+q-1}, t\right)+\mathcal{O}\left(h^{2}\right),
\end{aligned}
$$

where

$$
\begin{aligned}
& \omega_{0}^{(\alpha)}=\gamma_{1}(\alpha) g_{0}^{(\alpha)}, \quad \omega_{1}^{(\alpha)}=\gamma_{1}(\alpha) g_{1}^{(\alpha)}+\gamma_{0}(\alpha) g_{0}^{(\alpha)}, \\
& \omega_{k}^{(\alpha)}=\gamma_{1}(\alpha) g_{k}^{(\alpha)}+\gamma_{0}(\alpha) g_{k-1}^{(\alpha)}+\gamma_{-1}(\alpha) g_{k-2}^{(\alpha)}, \quad k \geq 2,
\end{aligned}
$$

in which

$$
\begin{aligned}
& \gamma_{1}(\alpha)=\frac{\alpha^{2}+3 \alpha+2}{12}, \quad \gamma_{0}(\alpha)=\frac{4-\alpha^{2}}{6}, \quad \gamma_{-1}(\alpha)=\frac{\alpha^{2}-3 \alpha+2}{12}, \\
& g_{0}^{(\alpha)}=1, \quad g_{k+1}^{(\alpha)}=\left(1-\frac{\alpha+1}{k+1}\right) g_{k}^{(\alpha)}, \quad k \geq 0 .
\end{aligned}
$$

Proposition 2.1 (See [15]). Let $\omega_{i}^{(\alpha)}, \alpha \in(1,2)$, be defined as in (2.3). Then the coefficients $\omega_{i}^{(\alpha)}$ satisfy the following properties:

$$
\begin{aligned}
& \omega_{0}^{(\alpha)}=\frac{\alpha^{2}+3 \alpha+2}{12}>0, \quad \omega_{1}^{(\alpha)}=\frac{-\alpha^{3}-5 \alpha^{2}-2 \alpha+8}{12}<0 \\
& \omega_{2}^{(\alpha)}=\frac{\alpha^{4}+6 \alpha^{3}+\alpha^{2}-24 \alpha+4}{24}, \quad 1 \geq \omega_{0}^{(\alpha)} \geq \omega_{3}^{(\alpha)} \geq \cdots \geq 0 \\
& \sum_{i=0}^{\infty} \omega_{i}^{(\alpha)}=0, \quad \sum_{i=0}^{n} \omega_{i}^{(\alpha)}<0, \quad n \geq 1 \\
& \omega_{0}^{(\alpha)}+\omega_{2}^{(\alpha)}>0 .
\end{aligned}
$$

Note that $\omega_{2}^{(\alpha)}$ may not be positive. 
Concerning the discretization in time, as done in [10], we take $t_{j+\frac{1}{2}}=t_{j}+\frac{\Delta t}{2}$ and consider the following central difference scheme:

$$
\frac{\partial u\left(t_{j+\frac{1}{2}}\right)}{\partial t}=\frac{u\left(x_{i}, t_{j+1}\right)-u\left(x_{i}, t_{j}\right)}{\Delta t}+\mathcal{O}\left(\Delta t^{2}\right)
$$

In order to approximate the integral in (2.1), we first decompose it at the subintervals arising from a partition of the integral interval $(1,2)$. Specifically, we divide the interval $(1,2)$ into $l$ uniform subintervals and denote by $\Delta \alpha$ the length of such subintervals. Then, the mid-point of each subinterval is given by $\alpha_{k}=1+\left(k-\frac{1}{2}\right) \Delta \alpha, k=1,2, \ldots, l$. Employing the mid-point quadrature rule, the integral term in (2.1) can be written as follows:

$$
\begin{aligned}
\int_{1}^{2} \rho(\alpha) \frac{\partial^{\alpha} u(x, t)}{\partial|x|^{\alpha}} d \alpha & =\sum_{k=1}^{l} \int_{1+(k-1) \Delta \alpha}^{1+k \Delta \alpha} \rho(\alpha) \frac{\partial^{\alpha} u(x, t)}{\partial|x|^{\alpha}} d \alpha \\
& =\sum_{k=1}^{l}\left[\rho\left(\alpha_{k}\right) \frac{\partial^{\alpha_{k}} u(x, t)}{\partial|x|^{\alpha_{k}}}\right] \Delta \alpha+\mathcal{O}\left(\Delta \alpha^{2}\right) .
\end{aligned}
$$

Combining (2.2), (2.5), (2.6), and (2.1), we obtain

$$
\begin{aligned}
& \frac{u\left(x_{i}, t_{j+1}\right)-u\left(x_{i}, t_{j}\right)}{\Delta t} \\
& =\Delta \alpha \sum_{k=1}^{l} \frac{\rho\left(\alpha_{k}\right) c\left(\alpha_{k}\right)}{h^{\alpha_{k}}}\left(\sum_{q=0}^{i} \omega_{q}^{\left(\alpha_{k}\right)} u\left(x_{i-q+1, t_{j+\frac{1}{2}}}\right)+\sum_{q=0}^{n-i+1} \omega_{q}^{\left(\alpha_{k}\right)} u\left(x_{i+q-1, t_{j+\frac{1}{2}}}\right)\right) \\
& \quad+f\left(x_{i}, t_{j+\frac{1}{2}}\right)+R_{i}^{j},
\end{aligned}
$$

where $R_{i}^{j}=\mathcal{O}\left(h^{2}+\Delta t^{2}+\Delta \alpha^{2}\right), 1 \leq i \leq n, 1 \leq j \leq m$.

Let $u_{i}^{j} \approx u\left(x_{i}, t_{j}\right)$ and $f_{i}^{j+\frac{1}{2}}=f\left(x_{i}, t_{j+\frac{1}{2}}\right)$. By omitting the small terms $R_{i}^{j}$ in (2.7), we arrive at the following difference finite scheme for solving (2.1):

$$
\begin{aligned}
u_{i}^{j+1}=u_{i}^{j}+ & \frac{\Delta t \Delta \alpha}{2} \sum_{k=1}^{l} \frac{\rho\left(\alpha_{k}\right) c\left(\alpha_{k}\right)}{h^{\alpha_{k}}}\left(\sum_{q=0}^{i} \omega_{q}^{\left(\alpha_{k}\right)}\left(u_{i-q+1}^{j+1}+u_{i-q+1}^{j}\right)\right. \\
& \left.+\sum_{q=0}^{n-i+1} \omega_{q}^{\left(\alpha_{k}\right)}\left(u_{i+q-1}^{j+1}+u_{i+q-1}^{j}\right)\right) \\
+\Delta t f_{i}^{j+\frac{1}{2}}, & 1 \leq i \leq n, 1 \leq j \leq m,
\end{aligned}
$$

with the initial condition $u_{i}^{0}=u_{0}\left(x_{i}\right)$, for $i=0,1, \ldots, n+1$, and the boundary condition $u_{0}^{j}=u\left(a, t_{j}\right), u_{n+1}^{j}=u\left(b, t_{j}\right)$, for $j=0,1, \ldots, m$. This numerical scheme has been proved to be unconditionally stable and to have a second-order convergence rate in both space and time directions in [1].

Let $u^{j}=\left(u_{1}^{j}, u_{2}^{j}, \ldots, u_{n}^{j}\right)^{T}, f^{j+\frac{1}{2}}=\left(f_{1}^{j+\frac{1}{2}}, f_{2}^{j+\frac{1}{2}}, \ldots, f_{n}^{j+\frac{1}{2}}\right)^{T}$, and let $I$ be the identity of size $n$. Thus, the above numerical scheme (2.8) can be written in the matrix form

$$
\left(I-A_{n}\right) u^{j+1}=\left(I+A_{n}\right) u^{j}+\Delta t f^{j+\frac{1}{2}},
$$

with

$$
A_{n}=\frac{\Delta t \Delta \alpha}{2} \sum_{k=1}^{l} \frac{\rho\left(\alpha_{k}\right) c\left(\alpha_{k}\right)}{h^{\alpha_{k}}} A_{n}\left(\alpha_{k}\right)
$$


where

$$
A_{n}\left(\alpha_{k}\right)=\left[\begin{array}{cccccc}
2 \omega_{1}^{\left(\alpha_{k}\right)} & \omega_{0}^{\left(\alpha_{k}\right)}+\omega_{2}^{\left(\alpha_{k}\right)} & \omega_{3}^{\left(\alpha_{k}\right)} & \ldots & \omega_{n-1}^{\left(\alpha_{k}\right)} & \omega_{n}^{\left(\alpha_{k}\right)} \\
\omega_{0}^{\left(\alpha_{k}\right)}+\omega_{2}^{\left(\alpha_{k}\right)} & 2 \omega_{1}^{\left(\alpha_{k}\right)} & \omega_{0}^{\left(\alpha_{k}\right)}+\omega_{2}^{\left(\alpha_{k}\right)} & \omega_{3}^{\left(\alpha_{k}\right)} & \cdots & \omega_{n-1}^{\left(\alpha_{k}\right)} \\
\vdots & \omega_{0}^{\left(\alpha_{k}\right)}+\omega_{2}^{\left(\alpha_{k}\right)} & 2 \omega_{1}^{\left(\alpha_{k}\right)} & \ddots & \ddots & \vdots \\
\vdots & \ddots & \ddots & \ddots & \ddots & \omega_{3}^{\left(\alpha_{k}\right)} \\
\omega_{n-1}^{\left(\alpha_{k}\right)} & \ddots & \ddots & \ddots & 2 \omega_{1}^{\left(\alpha_{k}\right)} & \omega_{0}^{\left(\alpha_{k}\right)}+\omega_{2}^{\left(\alpha_{k}\right)} \\
\omega_{n}^{\left(\alpha_{k}\right)} & \omega_{n-1}^{\left(\alpha_{k}\right)} & \cdots & \cdots & \omega_{0}^{\left(\alpha_{k}\right)}+\omega_{2}^{\left(\alpha_{k}\right)} & 2 \omega_{1}^{\left(\alpha_{k}\right)}
\end{array}\right]
$$

We can also write $A_{n}\left(\alpha_{k}\right)=A_{\alpha_{k}, n}+A_{\alpha_{k}, n}^{T}$, where

$$
A_{\alpha_{k}, n}=\left[\begin{array}{cccccc}
\omega_{1}^{\left(\alpha_{k}\right)} & \omega_{0}^{\left(\alpha_{k}\right)} & 0 & \cdots & 0 & 0 \\
\omega_{2}^{\left(\alpha_{k}\right)} & \omega_{1}^{\left(\alpha_{k}\right)} & \omega_{0}^{\left(\alpha_{k}\right)} & \ddots & \ddots & 0 \\
\vdots & \omega_{2}^{\left(\alpha_{k}\right)} & \omega_{1}^{\left(\alpha_{k}\right)} & \ddots & \ddots & \vdots \\
\vdots & \ddots & \ddots & \ddots & \ddots & \vdots \\
\omega_{n-1}^{\left(\alpha_{k}\right)} & \ddots & \ddots & \ddots & \omega_{1}^{\left(\alpha_{k}\right)} & \omega_{0}^{\left(\alpha_{k}\right)} \\
\omega_{n}^{(\alpha-1)} & \omega_{n-1}^{\left(\alpha_{k}\right)} & \cdots & \cdots & \omega_{2}^{\left(\alpha_{k}\right)} & \omega_{1}^{\left(\alpha_{k}\right)}
\end{array}\right]
$$

It is clear that $A_{n}\left(\alpha_{k}\right)$ is a symmetric Toeplitz matrix. As a consequence, the matrix $A_{n}=\left[A_{i j}\right]_{n \times n}=\left[a_{\mid i-j}\right]_{i, j=1}^{n}$ also possesses the symmetric Toeplitz structure, and the corresponding elements are

$$
a_{0}=2 \omega_{1}, \quad a_{1}=\omega_{0}+\omega_{2}, \quad a_{i}=\omega_{i+1}, \quad \text { for } i \geq 2,
$$

in which, by (2.10),

$$
\omega_{i}=\frac{\Delta t \Delta \alpha}{2} \sum_{k=1}^{l} \frac{\rho\left(\alpha_{k}\right) c\left(\alpha_{k}\right)}{h^{\alpha_{k}}} \omega_{i}^{\left(\alpha_{k}\right)}, \quad \text { for } i=0,1, \ldots, n .
$$

From Proposition 2.1, the next corollary immediately follows:

COROLLARY 2.2. The coefficients $\omega_{k}$ in (2.12) satisfy

$$
\begin{aligned}
& \omega_{0}>0, \quad \omega_{1}<0, \quad \text { and } \quad \omega_{k}>0, \quad k \geq 3, \\
& \omega_{0}+\omega_{2}>0, \\
& \sum_{k=0}^{\infty} \omega_{k}=0, \quad \sum_{k=0}^{n} \omega_{k}<0, \quad n \geq 1 .
\end{aligned}
$$

Lemma 2.3 (see [16]). The matrix $A_{n}$ defined in (2.10) is symmetric negative definite. For convenience, we rewrite the linear system in (2.9) as

$$
M_{n} u^{j+1}=b^{j},
$$

where

$$
M_{n}=I-A_{n}, \quad b^{j}=\left(I+A_{n}\right) u^{j}+\Delta t f^{j+\frac{1}{2}} .
$$

We stress that, due to Lemma 2.3, $M_{n}$ is of course a symmetric positive definite Toeplitz matrix. 
3. Spectral analysis of the coefficient matrices. In this section, we perform a spectral analysis of the coefficient matrix-sequence $\left\{M_{n}\right\}_{n}$. Specifically, we find its symbol and study its spectral distribution by using well-known tools for Toeplitz sequences. We will leverage the resulting spectral knowledge to introduce ad hoc preconditioners for solving equation (2.13).

Let us first introduce some basic definitions and results.

DEFINITION 3.1. Let $f \in L^{1}([-\pi, \pi])$, and let $\left\{f_{k}\right\}_{k \in \mathbb{Z}}$ be the sequence of its Fourier coefficients defined as

$$
f_{k}=\frac{1}{2 \pi} \int_{-\pi}^{\pi} f(\theta) e^{-i k \theta} d \theta, \quad k \in \mathbb{Z}
$$

Then the matrix-sequence $\left\{T_{n}\right\}_{n \in \mathbb{N}}$ with $T_{n}=\left[f_{i-j}\right]_{i, j=1}^{n}$ is called the sequence of Toeplitz matrices generated by $f$, which in turn is called the symbol of $\left\{T_{n}\right\}_{n \in \mathbb{N}}$, and $T_{n}$ is denoted by $T_{n}(f)$.

DEFINITION 3.2. The Wiener class is the set of functions $f(\theta)=\sum_{k=-\infty}^{\infty} f_{k} e^{i k \theta}$ such that $\sum_{k=-\infty}^{\infty}\left|f_{k}\right|<\infty$.

Note that the Wiener class forms a subalgebra of the continuous and $2 \pi$-periodic functions.

REMARK 3.3. Let $\left\{T_{n}\right\}_{n \in \mathbb{N}}$ be a Toeplitz sequence with $T_{n}=\left[f_{i-j}\right]_{i, j=1}^{n}$. If $\left\{f_{k}\right\}_{k \in \mathbb{Z}}$ is such that $\sum_{k=-\infty}^{\infty}\left|f_{k}\right|<\infty$, then the series $\sum_{k=-\infty}^{\infty} f_{k} e^{\mathrm{i} k \theta}$ converges uniformly in the infinity norm to a continuous and $2 \pi$-periodic function $f$ which belongs to the Wiener class and which is the symbol of $\left\{T_{n}\right\}_{n \in \mathbb{N}}$, i.e., $T_{n}=T_{n}(f)$, for all $n \in \mathbb{N}$. We continue with the definition of the spectral distribution in the sense of the eigenvalues.

DeFinition 3.4. Let $f:[a, b] \rightarrow \mathbb{C}$ be a measurable function defined on $[a, b] \subset \mathbb{R}$. Let $\mathcal{C}_{0}(\mathbb{C})$ be the set of continuous functions with compact support over $\mathbb{C}$, and let $\left\{\mathcal{A}_{n}\right\}_{n}$ be a sequence of matrices of size $n$ with eigenvalues $\lambda_{j}\left(\mathcal{A}_{n}\right), j=1, \ldots, n$. We say that $\left\{\mathcal{A}_{n}\right\}_{n}$ is distributed as the pair $(f,[a, b])$ in the sense of the eigenvalues, and we write

$$
\left\{\mathcal{A}_{n}\right\}_{n} \sim_{\lambda}(f,[a, b])
$$

if the following limit relation holds for all $F \in \mathcal{C}_{0}(\mathbb{C})$ :

$$
\lim _{n \rightarrow \infty} \frac{1}{n} \sum_{j=1}^{n} F\left(\lambda_{j}\left(\mathcal{A}_{n}\right)\right)=\frac{1}{b-a} \int_{a}^{b} F(f(t)) d t .
$$

REMARK 3.5. When $f$ is continuous, an informal interpretation of the limit relation (3.1) is that when the matrix-size is sufficiently large, the eigenvalues of $\mathcal{A}_{n}$ can be approximated by a sampling of $f$ on a uniform equispaced grid of the interval $[a, b]$. For Hermitian Toeplitz matrix-sequences, the following theorem due to Szegö, Tyrtyshnikov, etc., holds (see, e.g., [14]).

THEOREM 3.6. Let $f \in L^{1}([-\pi, \pi])$ be a real-valued function. Then,

$$
\left\{T_{n}(f)\right\}_{n} \sim_{\lambda}(f,[-\pi, \pi]) .
$$

We end this introductory part by recalling a property of the spectral norm of Toeplitz matrices and stating a relevant theorem from [3]. Given a square matrix $X$ of order $n$, we denote its spectral norm by $\|X\|$, i.e., the maximal singular value of $X\left(\|X\|=\max _{i=1, \ldots, n} \sigma_{i}(X)\right)$. This value coincides with the spectral radius in the case of a normal matrix, and we recall that every Hermitian matrix is also normal. Given a Toeplitz sequence $\left\{T_{n}(f)\right\}_{n \in \mathbb{N}}$ generated by $f$, it holds that (see [21, Corollary 3.5]):

$$
f \in L^{\infty}(-\pi, \pi] \Rightarrow\left\|T_{n}(f)\right\| \leq\|f\|_{\infty}, \quad \forall n \in \mathbb{N} .
$$


THEOREM 3.7 ([3, Cor. 2.8]). Let $\left\{\mathcal{A}_{n}\right\}_{n \in \mathbb{N}}$ be a matrix sequence with $\mathcal{A}_{n}=B_{n}+C_{n}$ and $B_{n}$ Hermitian, for all $n \in \mathbb{N}$. Assume that

- $\left\{B_{n}\right\}_{n \in \mathbb{N}} \sim_{\lambda}(f,[a, b])$,

- $\left\|C_{n}\right\|=o(1)$.

Then $\left\{\mathcal{A}_{n}\right\}_{n \in \mathbb{N}} \sim_{\lambda}(f,[a, b])$.

We are now ready to determine the symbol of our coefficient matrix-sequence. In that respect, we start to compute the symbol associated to $\left\{A_{\alpha_{k}, n}\right\}_{n \in \mathbb{N}}$.

PROPOSITION 3.8. Let $\alpha \in(1,2)$. The symbol associated to the matrix-sequence $\left\{A_{\alpha, n}\right\}_{n \in \mathbb{N}}$ belongs to the Wiener class, and its formal expression is given by

$$
\begin{aligned}
f_{\alpha}(\theta) & =\sum_{k=-1}^{\infty} \omega_{k+1}^{(\alpha)} e^{i k \theta} \\
& =\left[\frac{8-2 \alpha^{2}+\left(\alpha^{2}+3 \alpha+2\right) e^{-i \theta}+\left(\alpha^{2}-3 \alpha+2\right) e^{i \theta}}{12}\right]\left(1+e^{i(\theta+\pi)}\right)^{\alpha} .
\end{aligned}
$$

Proof. According to (2.11), we remind that $A_{\alpha, n}=\left[\omega_{i-j+1}^{(\alpha)}\right]_{i, j=1}^{n}$, with $\omega_{k}^{(\alpha)}=0$ for $k<0$. Then, by Definition 3.1, it holds that $A_{\alpha, n}=T_{n}\left(f_{\alpha}\right)$, where the generating function $f_{\alpha}$ is defined as

$$
f_{\alpha}(\theta)=\sum_{k=-1}^{\infty} \omega_{k+1}^{(\alpha)} e^{\mathrm{i} k \theta} .
$$

When $\alpha \in(1,2)$, it easy to see that $f_{\alpha}(\theta)$ lies in the Wiener class. In detail, from Proposition 2.1 we know that $\omega_{1}^{(\alpha)}=\frac{-\alpha^{3}-5 \alpha^{2}-2 \alpha+8}{12}<0, \omega_{2}^{(\alpha)}=\frac{\alpha^{4}+6 \alpha^{3}+\alpha^{2}-24 \alpha+4}{24}, \omega_{k}^{(\alpha)} \geq 0$, for $k \geq 0$ and $k \neq 1,2$, and $\omega_{k}^{(\alpha)}=0$, for $k<0$. Then

$$
\begin{aligned}
\sum_{k=-1}^{\infty}\left|\omega_{k+1}^{(\alpha)}\right| & =\sum_{\substack{k=-1 \\
k \neq 0,1}}^{\infty} \omega_{k+1}^{(\alpha)}+\left|\omega_{1}^{(\alpha)}\right|+\left|\omega_{2}^{(\alpha)}\right| \\
& =\sum_{\substack{k=-1 \\
k \neq 0,1}}^{\infty} \omega_{k+1}^{(\alpha)}+\frac{\alpha^{3}+5 \alpha^{2}+2 \alpha-8}{12}+\frac{\left|\alpha^{4}+6 \alpha^{3}+\alpha^{2}-24 \alpha+4\right|}{24}
\end{aligned}
$$

Again from Proposition 2.1, we obtain

$$
\begin{aligned}
\sum_{k=0}^{\infty} \omega_{k}^{(\alpha)}=0 & \Longleftrightarrow \sum_{\substack{k=-1 \\
k \neq 0,1}}^{\infty} \omega_{k+1}^{(\alpha)}=-\omega_{1}^{(\alpha)}-\omega_{2}^{(\alpha)} \\
& =-\frac{-\alpha^{3}-5 \alpha^{2}-2 \alpha+8}{12}-\frac{\alpha^{4}+6 \alpha^{3}+\alpha^{2}-24 \alpha+4}{24} \\
& =\frac{\alpha^{3}+5 \alpha^{2}+2 \alpha-8}{12}-\frac{\alpha^{4}+6 \alpha^{3}+\alpha^{2}-24 \alpha+4}{24}
\end{aligned}
$$

that is,

$$
\begin{aligned}
\sum_{k=-1}^{\infty}\left|\omega_{k+1}^{(\alpha)}\right|= & \frac{\alpha^{3}+5 \alpha^{2}+2 \alpha-8}{6} \\
& +\frac{\left|\alpha^{4}+6 \alpha^{3}+\alpha^{2}-24 \alpha+4\right|-\left(\alpha^{4}+6 \alpha^{3}+\alpha^{2}-24 \alpha+4\right)}{24}
\end{aligned}
$$


which shows that $\sum_{k=-1}^{\infty}\left|\omega_{k+1}^{(\alpha)}\right|$ converges for $\alpha \in(1,2)$. Then, thanks to Remark 3.3, $f_{\alpha}(\theta)$ belongs to the Wiener class. To obtain an explicit formula for the symbol $f_{\alpha}(\theta)$, we exploit the definition of $\omega_{k}^{(\alpha)}$ given in (2.3) involving the one of $g_{k+1}^{(\alpha)}$ in (2.4), that is,

$$
\begin{aligned}
f_{\alpha}(\theta)= & \sum_{k=0}^{\infty} \omega_{k}^{(\alpha)} e^{\mathrm{i}(k-1) \theta}=\frac{\alpha^{2}+3 \alpha+2}{12} g_{0}^{(\alpha)} e^{-\mathrm{i} \theta}+\left(\frac{\alpha^{2}+3 \alpha+2}{12} g_{1}^{(\alpha)}+\frac{4-\alpha^{2}}{6} g_{0}^{(\alpha)}\right) \\
& +\sum_{k=2}^{\infty}\left(\frac{\alpha^{2}+3 \alpha+2}{12} g_{k}^{(\alpha)}+\frac{4-\alpha^{2}}{6} g_{k-1}^{(\alpha)}+\frac{\alpha^{2}-3 \alpha+2}{12} g_{k-2}\right) e^{\mathrm{i}(k-1) \theta} \\
= & \frac{\alpha^{2}+3 \alpha+2}{12} \sum_{k=0}^{\infty}(-1)^{k}\left(\begin{array}{l}
\alpha \\
k
\end{array}\right) e^{\mathrm{i}(k-1) \theta}+\frac{4-\alpha^{2}}{6} \sum_{k=0}^{\infty}(-1)^{k}\left(\begin{array}{l}
\alpha \\
k
\end{array}\right) e^{\mathrm{i} k \theta} \\
& +\frac{\alpha^{2}-3 \alpha+2}{12} \sum_{k=0}^{\infty}(-1)^{k}\left(\begin{array}{l}
\alpha \\
k
\end{array}\right) e^{\mathrm{i}(k+1) \theta} \\
= & \frac{\alpha^{2}+3 \alpha+2}{12} e^{-\mathrm{i} \theta} \sum_{k=0}^{\infty}\left(\begin{array}{l}
\alpha \\
k
\end{array}\right) e^{\mathrm{i} k(\theta+\pi)}+\frac{4-\alpha^{2}}{6} \sum_{k=0}^{\infty}\left(\begin{array}{l}
\alpha \\
k
\end{array}\right) e^{\mathrm{i} k(\theta+\pi)} \\
& +\frac{\alpha^{2}-3 \alpha+2}{12} e^{\mathrm{i} \theta} \sum_{k=0}^{\infty} k(-1)^{k}\left(\begin{array}{l}
\alpha \\
k
\end{array}\right) e^{\mathrm{i} k(\theta+\pi)} .
\end{aligned}
$$

Applying the well-known binomial series

$$
(1+z)^{\alpha}=\sum_{k=0}^{\infty}\left(\begin{array}{l}
\alpha \\
k
\end{array}\right) z^{k}, \quad z \in \mathbb{Z},|z| \leq 1, \alpha>0,
$$

with $z=e^{\mathrm{i}(\theta+\pi)}$, we obtain

$$
f_{\alpha}(\theta)=\left[\frac{8-2 \alpha^{2}+\left(\alpha^{2}+3 \alpha+2\right) e^{-\mathrm{i} \theta}+\left(\alpha^{2}-3 \alpha+2\right) e^{\mathrm{i} \theta}}{12}\right]\left(1+e^{\mathrm{i}(\theta+\pi)}\right)^{\alpha},
$$

and the thesis is proven.

COROLlary 3.9. Let $\alpha \in(1,2)$. The symbol associated to the matrix-sequence $\left\{A_{n}(\alpha)=A_{\alpha, n}+A_{\alpha, n}^{T}\right\}_{n \in \mathbb{N}}$ belongs to the Wiener class, and its formal expression is given by

$$
g_{\alpha}(\theta)=f_{\alpha}(\theta)+f_{\alpha}(-\theta),
$$

where $f_{\alpha}$ is defined as in (3.3).

Remark 3.10. A similar reasoning to the one made in [7, Proposition 4] proves that the function $g_{\alpha}(\theta)$ is a nonpositive function with a single zero at 0 of order $\alpha$.

COROLLARY 3.11. Let $A_{n}$ be the matrix defined in (2.10) and assume that $h^{\Delta \alpha}=o(1)$. Then,

$$
\left\{\frac{h^{\alpha_{l}}}{\Delta t \Delta \alpha} A_{n}\right\}_{n \in \mathbb{N}} \sim_{\lambda}\left(c_{l} g_{\alpha_{l}}(\theta),[0, \pi]\right), \quad \text { where } \quad c_{l}=\frac{\rho\left(\alpha_{l}\right) c\left(\alpha_{l}\right)}{2} .
$$

Proof. From (2.10),

$$
A_{n}=\Delta t \Delta \alpha\left(\frac{c_{1}}{h^{\alpha_{1}}} A_{n}\left(\alpha_{1}\right)+\frac{c_{2}}{h^{\alpha_{2}}} A_{n}\left(\alpha_{2}\right)+\frac{c_{3}}{h^{\alpha_{3}}} A_{n}\left(\alpha_{3}\right)+\cdots+\frac{c_{l}}{h^{\alpha_{l}}} A_{n}\left(\alpha_{l}\right)\right),
$$


with $c_{k}=\frac{\rho\left(\alpha_{k}\right) c\left(\alpha_{k}\right)}{2}$. Then,

$$
\begin{aligned}
\frac{h^{\alpha_{l}}}{\Delta t \Delta \alpha} A_{n}=c_{1} h^{\alpha_{l}-\alpha_{1}} A_{n}\left(\alpha_{1}\right)+c_{2} h^{\alpha_{l}-\alpha_{2}} A_{n}\left(\alpha_{2}\right) & +\cdots \\
& +c_{l-1} h^{\alpha_{l}-\alpha_{l-1}} A_{n}\left(\alpha_{l-1}\right)+c_{l} A_{n}\left(\alpha_{l}\right) \\
=c_{1} h^{\Delta \alpha(l-1)} A_{n}\left(\alpha_{1}\right)+c_{2} h^{\Delta \alpha(l-2)} & A_{n}\left(\alpha_{2}\right)+\cdots \\
& +c_{l-1} h^{\Delta \alpha} A_{n}\left(\alpha_{l-1}\right)+c_{l} A_{n}\left(\alpha_{l}\right) .
\end{aligned}
$$

Since $1<\alpha_{1}<\alpha_{2}<\cdots<\alpha_{l}<2$, we have that $\alpha_{l}-\alpha_{k}>0$, for $k=1, \ldots, l-1$. As a consequence, by using (3.2) and the hypothesis $h^{\Delta \alpha}=o(1)$, it holds that

$$
\left\|c_{k} h^{\alpha_{l}-\alpha_{k}} A_{n}\left(\alpha_{k}\right)\right\| \leq c_{k} h^{\alpha_{l}-\alpha_{k}}\left\|g_{\alpha_{k}}\right\|_{\infty} \rightarrow 0,
$$

and this yields the thesis by applying Theorem 3.7 with the setting $B_{n}=c_{l} A_{n}\left(\alpha_{l}\right)$ and $C_{n}=\frac{h^{\alpha_{l}}}{\Delta t \Delta \alpha} A_{n}-B_{n}$.

Similarly, for the matrix $M_{n}$, we prove the following spectral result:

COROLlary 3.12. For the matrix $M_{n}$ defined in (2.13), when $h^{\alpha_{l}}=o(\Delta t \Delta \alpha)$ and $h^{\Delta \alpha}=o(1)$, it holds that

$$
\left\{\frac{h^{\alpha_{l}}}{\Delta t \Delta \alpha} M_{n}\right\}_{n \in \mathbb{N}} \sim_{\lambda}\left(-c_{l} g_{\alpha_{l}}(\theta),[0, \pi]\right), \quad \text { where } \quad c_{l}=\frac{\rho\left(\alpha_{l}\right) c\left(\alpha_{l}\right)}{2} .
$$

Proof. The thesis follows from

$$
\frac{h^{\alpha_{l}}}{\Delta t \Delta \alpha} M_{n}=\frac{h^{\alpha_{l}}}{\Delta t \Delta \alpha} I-\frac{h^{\alpha_{l}}}{\Delta t \Delta \alpha} A_{n}
$$

and applying Theorem 3.7 with $B_{n}=-\frac{h^{\alpha_{l}}}{\Delta t \Delta \alpha} A_{n}$ and $C_{n}=\frac{h^{\alpha} l}{\Delta t \Delta \alpha} I$. Indeed, by Corollary 3.11 it holds that $\left\{-\frac{h^{\alpha_{l}}}{\Delta t \Delta \alpha} A_{n}\right\}_{n \in \mathbb{N}} \sim_{\lambda}\left(-c_{l} g_{\alpha_{l}}(\theta),[0, \pi]\right)$. Furthermore, under the stated hypothesis that $h^{\alpha_{l}}=o(\Delta t \Delta \alpha)$, the remaining term $\frac{h^{\alpha_{l}}}{\Delta t \Delta \alpha} I$ is such that $\left\|\frac{h^{\alpha_{l}}}{\Delta t \Delta \alpha} I\right\|=$ $\frac{h^{\alpha} l}{\Delta t \Delta \alpha}=o(1)$, and the thesis is proven.

In the following we discuss three more issues:

- the case where the condition $h^{\Delta \alpha}=o(1)$ is not satisfied;

- the spectral analysis of the preconditioned matrix-sequences when using the Laplacianstyle preconditioning described by the formula

$$
L_{n}=I-\frac{\Delta t \Delta \alpha}{2} \sum_{k=1}^{l} \frac{\rho\left(\alpha_{k}\right) c\left(\alpha_{k}\right)}{h^{\alpha_{k}}} A_{n}(2) ;
$$

- the expected performances of a generic $\tau$-preconditioning in our context.

Under the assumption that $h^{\Delta \alpha} \sim 1$ (for instance, when $\Delta \alpha=h^{\gamma}(1+o(1)$ ) with $\gamma$ being a positive constant independent of $h$, we have $\lim _{h \rightarrow 0} h^{\Delta \alpha}=1$ ), by assuming that $h^{\alpha_{l}}=o(\Delta t \Delta \alpha)$, as already observed, we deduce that the two matrix-sequences

$$
\left\{\frac{h^{\alpha_{l}}}{\Delta t \Delta \alpha} M_{n}\right\}_{n \in \mathbb{N}}=\left\{\frac{h^{\alpha_{l}}}{\Delta t \Delta \alpha} I-\frac{h^{\alpha_{l}}}{\Delta t \Delta \alpha} A_{n}\right\}_{n \in \mathbb{N}}
$$

and

$$
\left\{-\frac{h^{\alpha_{l}}}{\Delta t \Delta \alpha} A_{n}\right\}_{n \in \mathbb{N}}
$$




\section{ETNA}

Kent State University and

Johann Radon Institute (RICAM)

share the same eigenvalue distribution, if any, since $\left\{\frac{h^{\alpha} l}{\Delta t \Delta \alpha} I\right\}_{n \in \mathbb{N}}$ is spectrally clustered at zero.

Now, $-A_{n}\left(\alpha_{k}\right)$ is the Toeplitz matrix of order $n$ generated by the nonnegative function $-g_{\alpha_{k}}$ having a unique zero at $\theta=0$ of order $\alpha_{k}$, and hence its minimal eigenvalue is asymptotically given by $h^{\alpha_{k}}$ according to [4, 23]. Thus, $-h^{\alpha_{l}-\alpha_{k}} A_{n}\left(\alpha_{k}\right)$ has a minimal eigenvalue tending to zero exactly as $h^{\alpha_{l}}$, independently of $k$. Consequently,

$$
-\frac{h^{\alpha_{l}}}{\Delta t \Delta \alpha} A_{n}=-\sum_{k=1}^{l} c_{k} h^{\alpha_{l}-\alpha_{k}} A_{n}\left(\alpha_{k}\right)
$$

can be regarded as a Toeplitz matrix generated by a function which is nonnegative and with a unique zero at $\theta=0$ of order $\alpha_{l}=2-\frac{1}{2} \Delta \alpha$. This conclusion is supported numerically and gives a strong theoretical motivation for choosing $-A_{n}(2)$, which is a standard tridiagonal Laplacian whose symbol is $2-2 \cos (\theta)$, as the main ingredient for the proposed preconditioning. In fact, by [22], we know that the eigenvalues of $T_{n}^{-1}(g) T_{n}(f)$, for $f, g \geq 0$, behave like a sampling of $f / g$ in its domain of definition, and hence, in our setting, the zero of the original matrix has order $\alpha_{l}=2-\frac{1}{2} \Delta \alpha$, and the symbol of the preconditioner has a zero of order 2 . It follows that our $f / g$ has a positive range, and hence, as numerically observed, it is optimal in the sense that the number of expected iterations does not grow with the matrix-size $n$.

Regarding the performances of a generic $\tau$-preconditioning, as described in [24], for symmetric Toeplitz matrices with real coefficients, we remind that the (real) $\tau$-algebra allows one to obtain approximations of the original coefficient matrix showing a correction with a slightly lower rank when compared with the classical circulant algebra: this slightly smaller rank correction is reflected in a slightly smaller number of preconditioned conjugate gradient (PCG) iterations as reported in the numerical tests.

4. Numerical results. This section is devoted to the numerical validation of the results obtained in Section 3. We start by numerically verifying the following two relations:

$$
\begin{aligned}
\left\{A_{n}\left(\alpha_{l}\right)\right\}_{n \in \mathbb{N}} & \sim_{\lambda}\left(g_{\alpha_{l}}(\theta),[0, \pi]\right), \\
\left\{\frac{h^{\alpha_{l}}}{\Delta t \Delta \alpha} A_{n}\right\}_{n \in \mathbb{N}} & \sim_{\lambda}\left(c_{l} g_{\alpha_{l}}(\theta),[0, \pi]\right), \quad \text { where } c_{l}=\frac{\rho\left(\alpha_{l}\right) c\left(\alpha_{l}\right)}{2}
\end{aligned}
$$

In light of Remark 3.5, this is done by comparing the eigenvalues of $A_{n}\left(\alpha_{l}\right)$ and $A_{n}$ (properly scaled) with a uniform sampling of $g_{\alpha_{l}}(\theta)$ (properly scaled as well) on $[0, \pi]$. In Figures 4.1(a)(c), where we fix $l=2$ and vary $n \in\{100,500,1000\}$, it is observed that as $n$ increases, the sampling of the generating function $c_{l} g_{\alpha_{l}}(\theta)$ becomes closer and closer to the eigenvalues of $\frac{h^{\alpha_{l}}}{\Delta t \Delta \alpha} A_{n}$. From Figure 4.1(d), we see that the matching between $c_{l} g_{\alpha_{l}}(\theta)$ and $\frac{h^{\alpha} l}{\Delta t \Delta \alpha} A_{n}$ is already accurate for $n=100$. Similar results are obtained in Figure 4.2, which refers to the case where $l=5$. Note that in this case the eigenvalues of $\frac{h^{\alpha} l}{\Delta t \Delta \alpha} A_{n}$ approach the sampling of $c_{l} g_{\alpha_{l}}(\theta)$ less quickly, and this is why here the largest tested dimension is $n=10000$. The reason of the observed different behavior lies in the fact that for larger $l$, but still fixed and independent of $n$, the number of corrections terms increases: hence, for a large $l$, the spectral distribution result in (4.1) still holds asymptotically, but we need a larger size $n$ for observing the asymptotical relation (4.1) in practice. 


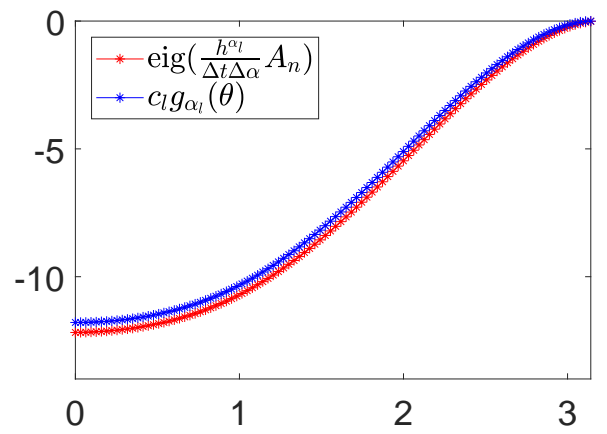

(a) $n=100$.

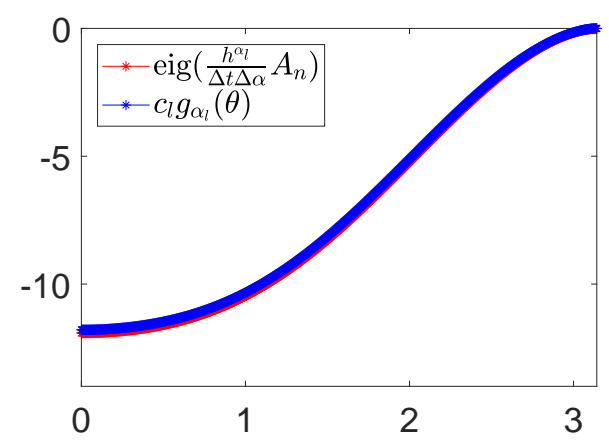

(c) $n=1000$.

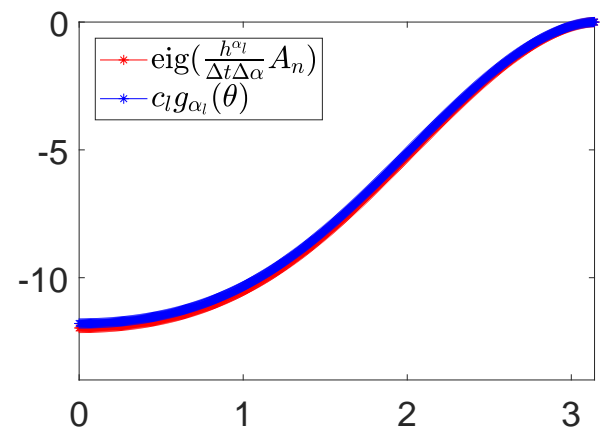

(b) $n=500$.

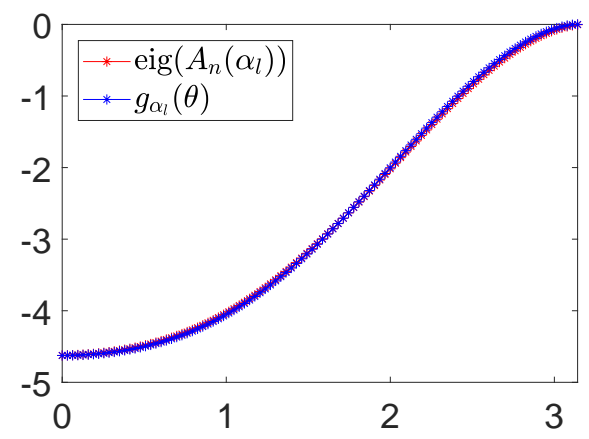

(d) $n=100$.

FIG. 4.1. (a)-(c) Comparison between the symbol $c_{l} g_{\alpha_{l}}(\theta)$ and eig $\left(\frac{h^{\alpha_{l}}}{\Delta t \Delta \alpha} A_{n}\right)$, for $l=2$ and $n=100,500,1000$. (d) Comparison between the symbol $g_{\alpha_{l}}(\theta)$ and eig $\left(A_{n}\left(\alpha_{l}\right)\right)$, for $l=2$ and $n=100$.

We now discuss the performances of the PCG method when applied to the following example taken from [16]: assume that in the problem equation (2.1), we set

$$
\begin{aligned}
u(x, 0) & =x^{2}(1-x)^{2}, \quad \rho(\alpha)=-2 \Gamma(5-\alpha) \cos \left(\frac{\alpha \pi}{2}\right), \\
f(x, t) & =e^{t} x^{2}(1-x)^{2}-\int_{1}^{2} \rho(\alpha) \frac{\partial^{\alpha} u(x, t)}{\partial|x|^{\alpha}} d \alpha \\
& =e^{t} x^{2}(1-x)^{2}-e^{t}\left[f_{1}(x)+f_{1}(1-x)\right]
\end{aligned}
$$

where

$$
\begin{array}{r}
f_{1}(x)=\Gamma(5) \frac{1}{\ln x}\left(x^{3}-x^{2}\right)-2 \Gamma(4)\left[\frac{1}{\ln x}\left(3 x^{2}-2 x\right)-\frac{1}{(\ln x)^{2}}\left(x^{2}-x\right)\right] \\
+\Gamma(3) \frac{1}{\ln x}\left[6 x-2-\frac{5 x}{\ln x}+\frac{3}{\ln x}+\frac{2 x}{(\ln x)^{2}}-\frac{2}{(\ln x)^{2}}\right] .
\end{array}
$$

The exact solution for this problem is

$$
u(x, t)=e^{t} x^{2}(1-x)^{2}, \quad \text { for }(x, t) \in[0,1] \times[0,1] .
$$

As stopping criterion for the PCG method, we consider

$$
\frac{\left\|r^{(k)}\right\|_{2}}{\left\|r^{(0)}\right\|_{2}}<10^{-8}
$$




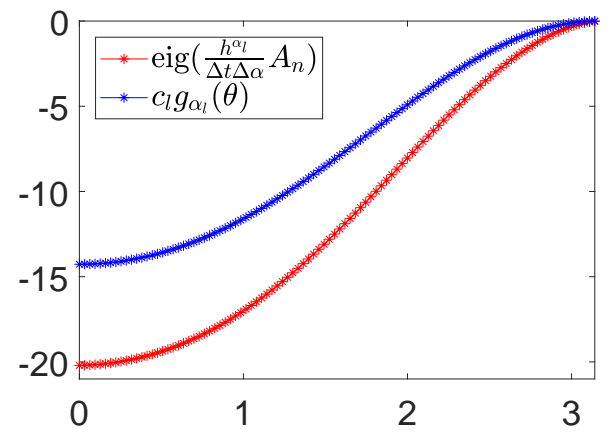

(a) $n=100$.

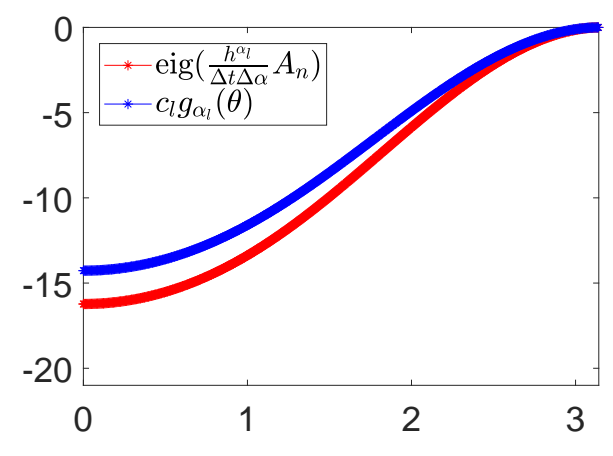

(c) $n=10000$

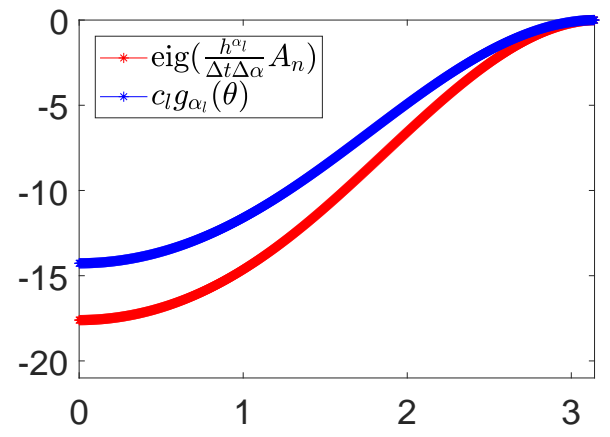

(b) $n=1000$.

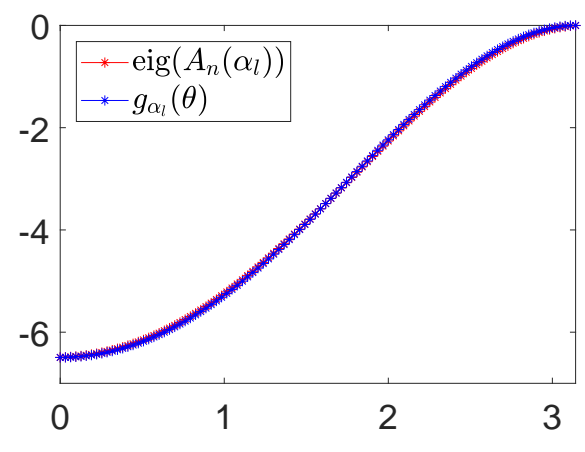

(d) $n=100$.

FIG. 4.2. (a)-(c) Comparison between the symbol $c_{l} g_{\alpha_{l}}(\theta)$ and eig $\left(\frac{h^{\alpha} l}{\Delta t \Delta \alpha} A_{n}\right)$, for $l=5$, and $n=$ $100,1000,1000$. (d) Comparison between the symbol $g_{\alpha_{l}}(\theta)$ and eig $\left(A_{n}\left(\alpha_{l}\right)\right)$, for $l=5$ and $n=100$.

where $r^{(k)}$ represents the residual vector after $k$ iterations. In Tables 4.1 and 4.2, the Laplacianlike and $\tau$-preconditioners discussed in Section 3 are compared with the Strang circulant proposed in [16], where the comparison is given both in terms of iterations and CPU times. In all tables, by "Iter" we mean the average number of iterations after 10 time-steps and by "CPU" the corresponding average timings in seconds. We also report the $L_{2}$-norm error defined as

$$
E_{2}(h)=\|u-\tilde{u}\|_{L_{2}}=\sqrt{h \sum_{j=1}^{n}\left(u_{j}-\tilde{u_{j}}\right)^{2}},
$$

where $u$ and $\tilde{u}$ are the numerical and exact solutions at the last considered time step, respectively. Since for all tested preconditioners the obtained errors are comparable, we only show the one referring to the Laplacian preconditioner.

Tables 4.1 and 4.2 refer to the case of fixed $l=5$ and to the case where $l=n$, respectively. We note that, independently of the chosen $l$, both $\tau$ - and Strang circulant preconditioners outperform the Laplacian one even though the cost of a Laplacian solver increases only linearly with the dimension. Moreover, in line with the theory, for the last two tested sizes $\left(2^{11}, 2^{12}\right)$, the $\tau$-preconditioning provides a slightly smaller number of iterations than the circulant, which translates into lower CPU timings. This result for the latter agrees with the rank analysis in [24]. We expect that for larger sizes the discrepancy between the circulant and the $\tau$-preconditioning becomes more evident and favorable to the latter approach. Having in mind the extension to multidimensional problems, we stress that the $\tau$-proposal is expected to be a better option than the circulant approach since it is well-known that multilevel circulant preconditioning 


\section{ETNA}

Kent State University and

Johann Radon Institute (RICAM)

cannot ensure a superlinear convergence character of the PCG due to the negative results in [26], while it is worth stressing that the computational cost of ad hoc Laplacian solvers in a multidimensional context is still linear with respect to the matrix-size.

TABLE 4.1

$P C G$ method performances with three different preconditioners. Here $l=5$.

\begin{tabular}{c|cc|cc|cc|c}
\multirow{2}{*}{$n=m$} & \multicolumn{2}{|c|}{ Strang circulant } & \multicolumn{2}{|c|}{$\tau$-preconditioner } & \multicolumn{2}{|c|}{ Laplacian } & \multirow{2}{*}{$E_{2}(h)$} \\
\cline { 2 - 7 } & Iter & CPU(s) & Iter & CPU(s) & Iter & CPU(s) & \\
\hline $2^{4}$ & 4.0 & 0.0000 & 4.0 & 0.0003 & 7.2 & 0.0001 & $1.37 \mathrm{e}-3$ \\
$2^{5}$ & 4.0 & 0.0000 & 4.0 & 0.0003 & 8.1 & 0.0001 & $3.49 \mathrm{e}-4$ \\
$2^{6}$ & 4.0 & 0.0001 & 4.0 & 0.0004 & 7.2 & 0.0002 & $8.66 \mathrm{e}-5$ \\
$2^{7}$ & 4.0 & 0.0003 & 4.0 & 0.0007 & 7.1 & 0.0006 & $2.13 \mathrm{e}-5$ \\
$2^{8}$ & 4.0 & 0.0007 & 4.0 & 0.0012 & 7.1 & 0.0011 & $5.23 \mathrm{e}-6$ \\
$2^{9}$ & 4.0 & 0.0059 & 4.0 & 0.0066 & 7.2 & 0.0077 & $1.28 \mathrm{e}-6$ \\
$2^{10}$ & 4.0 & 0.0166 & 3.8 & 0.0180 & 7.3 & 0.0284 & $3.15 \mathrm{e}-7$ \\
$2^{11}$ & 3.9 & 0.0608 & 3.0 & 0.0484 & 7.1 & 0.1067 & $7.76 \mathrm{e}-8$ \\
$2^{12}$ & 3.7 & 0.2322 & 3.1 & 0.1924 & 7.1 & 0.4326 & $1.92 \mathrm{e}-8$
\end{tabular}

TABLE 4.2

$P C G$ method performances with three different preconditioners. Here $l=n$.

\begin{tabular}{c|cc|cc|cc|c}
\multirow{2}{*}{$n=m$} & \multicolumn{2}{|c|}{ Strang circulant } & \multicolumn{2}{c|}{$\tau$-preconditioner } & \multicolumn{2}{|c|}{ Laplacian } & \multirow{2}{*}{$E_{2}(h)$} \\
\cline { 2 - 7 } & Iter & CPU(s) & Iter & CPU(s) & Iter & CPU(s) & \\
\hline $2^{4}$ & 4.0 & 0.0000 & 4.0 & 0.0003 & 7.1 & 0.0001 & $1.37 \mathrm{e}-3$ \\
$2^{5}$ & 4.0 & 0.0000 & 4.0 & 0.0003 & 8.1 & 0.0001 & $3.49 \mathrm{e}-4$ \\
$2^{6}$ & 4.0 & 0.0001 & 4.0 & 0.0004 & 7.1 & 0.0002 & $8.66 \mathrm{e}-5$ \\
$2^{7}$ & 4.0 & 0.0003 & 4.0 & 0.0008 & 7.1 & 0.0005 & $2.13 \mathrm{e}-5$ \\
$2^{8}$ & 4.0 & 0.0006 & 4.0 & 0.0013 & 7.1 & 0.0008 & $5.23 \mathrm{e}-6$ \\
$2^{9}$ & 4.0 & 0.0052 & 4.0 & 0.0065 & 7.1 & 0.0078 & $1.28 \mathrm{e}-6$ \\
$2^{10}$ & 4.0 & 0.0168 & 4.0 & 0.0189 & 6.7 & 0.0271 & $3.15 \mathrm{e}-7$ \\
$2^{11}$ & 4.0 & 0.0631 & 3.1 & 0.0500 & 6.1 & 0.0918 & $7.76 \mathrm{e}-8$ \\
$2^{12}$ & 4.0 & 0.2516 & 3.1 & 0.1984 & 5.1 & 0.3097 & $1.92 \mathrm{e}-8$
\end{tabular}

5. Conclusions and open problems. In this work, we have examined the numerical solution of a 1D distributed-order space-fractional diffusion equation. Discretizing the given problem by means of an implicit finite difference scheme based on the shifted GrünwaldLetnikov formula, the resulting linear systems show a Toeplitz structure. Then, by using wellknown spectral tools for Toeplitz sequences, we have computed and studied the corresponding symbol, describing its asymptotic eigenvalue distribution as the matrix size diverges. The spectral analysis has been discussed under different assumptions, with the aim of estimating the intrinsic asymptotic ill-conditioning of the involved matrices. The obtained results suggested to precondition the involved linear systems with either a Laplacian-like preconditioner or with more general $\tau$-preconditioners in the framework of the PCG method. As future immediate steps, we think that these strategies could be explored in the more challenging multilevel setting (for $2 \mathrm{D}$ or $3 \mathrm{D}$ problems, taking also into consideration the literature $[10,11,28]$ ), while a more technical analysis of the spectral features of the preconditioned matrices has to be provided especially when $h^{\Delta \alpha} \sim 1$. 
Having in mind the extension to multidimensional problems, we stress three aspects:

- the numerical approximation by local methods of a $d$-dimensional version of (2.1) with $d \geq 2$ leads to $d$-level Toeplitz matrix-sequences, and, from a spectral viewpoint, all the machinery for analyzing the extremal eigenvalues and the global distribution of the spectrum of such matrix-sequences is available (see [13] and references therein);

- the considered spectral tools can be adapted in an elegant manner to the case of a variable coefficient $d$-dimensional version of (2.1) via the (asymptotic) theory of $d$ level Generalized Locally Toeplitz matrix-sequences (see $[12,13]$ and the references therein);

- from an algorithmic viewpoint, the $\tau$-proposal is expected to be a better option than the circulant proposal in a $d$-dimensional setting, $d \geq 2$, since it is well-known that multilevel circulant preconditioning cannot ensure a superlinear convergence character of the PCG due to the negative results in [26], while it is worth stressing that the computational cost of ad hoc Laplacian solvers in a multidimensional context is still linear with respect to the matrix-size.

Finally we mention that, as discussed in [25, Section 3], the spectral tools used in this setting are convenient also for the study of the stability analysis of the numerical methods for timedependent differential equations. Such a topic is an interesting challenge also in case of fractional-differential equations (see, e.g., [27]) and will be the subject of future investigations.

Acknowledgment. The first and second authors are member of the INdAM research group GNCS. The work of the first author was partly supported by the GNCS-INdAM Young Researcher Project 2020 titled "Numerical methods for image restoration and cultural heritage deterioration".

\section{REFERENCES}

[1] M. ABBASZADEH, Error estimate of second-order finite difference scheme for solving the Riesz space distributed-order diffusion equation, Appl. Math. Lett., 88 (2019), pp. 179-185.

[2] J. BAI AND X. FENG, Fractional-order anisotropic diffusion for image denoising, IEEE Trans. Image Process., 16 (2007), pp. 2492-2502.

[3] G. BARBARINO AND S. SeRRA-CAPIZZANO, Non-Hermitian perturbations of Hermitian matrix-sequences and applications to the spectral analysis of the numerical approximation of partial differential equations, Numer. Linear Algebra Appl., 27 (2020), Art. e2286, 31 pages.

[4] A. BÖTTCHER AND S. GRUDSKY, On the condition numbers of large semi-definite Toeplitz matrices, Linear Algebra Appl., 279 (1998), pp. 285-301.

[5] M. CAPUTO, Diffusion with space memory modelled with distributed order space fractional differential equations, Ann. Geophys., 46 (2003), pp. 223-234.

[6] - Mean fractional-order-derivatives differential equations and filters, Ann. Univ. Ferrara Sez. VII (N.S.), 41 (1995), pp. 73-84.

[7] M. Donatelli, M. Mazza, and S. Serra-Capizzano, Spectral analysis and structure-preserving preconditioners for fractional diffusion equations, J. Comput. Phys., 307 (2016), pp. 262-279.

[8] - Spectral analysis and multigrid methods for finite volume approximations of space-fractional diffusion equations, SIAM J. Sci. Comput., 40 (2018), pp. A4007-A4039.

[9] D. Del-Castillo-Negrete, B. A. Carreras, and V. E. Lynch, Fractional diffusion in plasma turbulence, Phys. Plasmas, 11 (2004), pp. 3854-3864.

[10] W. FAN AND F. LIU, A numerical method for solving the two-dimensional distributed order space-fractional diffusion equation on an irregular convex domain, Appl. Math. Lett., 77 (2018), pp. 114-121.

[11] S. GuO, L. MEI, Z. ZHANG, AND Y. JIANG, Finite difference/spectral-Galerkin method for a two-dimensional distributed-order time-space fractional reaction-diffusion equation, Appl. Math. Lett., 85 (2018), pp. 157163.

[12] C. Garoni And S. Serra Capizzano, Generalized Locally Toeplitz Sequences: Theory and Applications. Vol. I, Springer, Cham, 2017.

[13] — Generalized Locally Toeplitz Sequences: Theory and Applications. Vol. II, Springer, Cham, 2018.

[14] U. Grenander And G. SZEgö, Toeplitz, Forms and Their Applications, 2nd ed., Chelsea, New York, 1984. 
[15] Z. HAO, Z. SUN, AND W. CAO, A fourth-order approximation of fractional derivatives with its applications, J. Comput. Phys., 281 (2015), pp. 787-805.

[16] X. Huang, Z. W. Fang, H. W. Sun, And C. H. Zhang, A circulant preconditioner for the Riesz distributedorder space-fractional diffusion equations, Linear and Multilinear Algebra, (2020), pp. 1-16, published online, https://doi.org/10.1080/03081087.2020.1823309.

[17] T. LANGlands, B. HeNRY, AND S. WEARNE, Fractional cable equation models for anomalous electrodiffusion in nerve cells: infinite domain solutions, J. Math. Biol., 59 (2009), pp. 761-808.

[18] J. Li, F. LIU, L. FENG, AND I. TURNER, A novel finite volume method for the Riesz space distributed order diffusion equation, Comput. Math. Appl., 74 (2017), pp. 772-783.

[19] H. Moghaderi, M. Dehghan, M. Donatelli, And M. Mazza, Spectral analysis and multigrid preconditioners for two-dimensional space-fractional diffusion equations, J. Comput. Phys., 350 (2017), pp. 992-1011.

[20] M. RABERTO, E. SCALAS, AND F. MAINARDI, Waiting-times and returns in high-frequency financial data: an empirical study, Phys. A, 314 (2002), pp. 749-755.

[21] S. SERRA-CAPIZZANO, More inequalities and asymptotics for matrix-valued linear positive operators: the noncommutative case, in Toeplitz Matrices and Singular Integral Equations, A. Böttcher, I. Gohberg, and P. Junghanns, eds., Oper. Theory. Adv. Appl., 135, Birkhäuser, Basel, 2002, pp. 293-315.

[22] _ New PCG based algorithms for the solution of Hermitian Toeplitz systems, Calcolo, 32 (1995), pp. 153-176.

[23] - On the extreme eigenvalues of Hermitian (block) Toeplitz matrices, Linear Algebra Appl., 270 (1998), pp. 109-129.

[24] - Toeplitz preconditioners constructed from linear approximation processes, SIAM J. Matrix Anal. Appl., 20 (1999), pp. 446-465.

[25] — - The GLT class as a generalized Fourier analysis and applications, Linear Algebra Appl., 419 (2006), pp. 180-233.

[26] S. SERRA-CAPIZZANO AND E. TYRTYSHNIKOV, Any circulant-like preconditioner for multilevel matrices is not superlinear, SIAM J. Matrix Anal. Appl., 21 (1999), pp. 431-439.

[27] R. Scherer, S. L. Kalla, Y. TANG, AND J. HuAng, The Grünwald-Letnikov method for fractional differential equations, Comput. Math. Appl., 62 (2011), pp. 902-917.

[28] X. ZHENG, H. LIU, H. WANG, AND H. FU, An efficient finite volume method for nonlinear distributed-order space-fractional diffusion equations in three space dimensions, J. Sci. Comp., 80 (2019), pp. 1395-1418. 